\title{
Delivery Long-Term Care Support Services and Care Recipients' Health Outcomes: Synthesis of Current Evidence
}

\section{Rashmita Basu*}

Baylor Scott and White Health Temple, TX 76502, USA

*Corresponding author: Rashmita Basu, Baylor Scott and White Health Temple, TX 76502, USA, Tel: 2547241172; E-mail: rashmita.basu@bswhealth.org

Received date: August 31, 2017; Accepted date: September 22, 2017; Published date: September 30, 2017

Copyright: (c) 2017 Basu R. This is an open-access article distributed under the terms of the Creative Commons Attribution License, which permits unrestricted use, distribution, and reproduction in any medium, provided the original author and source are credited.

\begin{abstract}
The combined impacts of population aging and longer life expectancies contribute to a surge in the number of older adults with physical and cognitive impairment. More than 12 million people (about 6.7 million are elderly aged over 65 years) in the U.S. need long-term care services and supports (LTSS) and this number is projected to be over 27 million by 2050. LTSS refers to a broad range of services to provide assistance over prolonged period of time due to physical or mental disability. People generally receive LTSS in a variety of settings- informally from friends and family or formal home and community based care or institutional care (such as nursing home). A major challenge facing the country is how best to meet the growing needs for LTSS in most homelike- setting and least institutional setting and to achieve best possible health outcomes. To address this challenge the evaluation of relative effectiveness of the use of informal and formal LTSS on care recipients' physical, emotional and mental health outcomes is imperative. The goals of this brief review are 1) to investigate existing evidence on the benefits of the use of informal and formal LTSS on the older adults' physical and mental health outcomes and 2) potential areas for future research on empirical evidence of the effectiveness of the use of informal and formal LTSS influencing care recipients' health outcomes.
\end{abstract}

Keywords: Long-term care support services; Informal care for formal care; Patients physical and mental health

\section{Introduction}

By 2050, one-fifth of the total U.S. population will be 65 years or older and the number of people aged 85 years or older will also grow faster over the next few decades [1]. This rising growth in the elderly population will lead to a surge in the number of elderly with physical and cognitive impairment. Physical and cognitive limitations are likely to restrict the elderly's ability to perform daily living activities independently in home or community-based settings. If the prevalence of cognitive impairment or loss of functional abilities continues to increase, the number of elderly in need for long-term care and support services (LTSS) will sharply increase in coming decades. More than 12 million people (about 6.7 million are elderly aged over 65 years) in the U.S. need LTSS and this number is projected to be over 27 million by 2050 [2]. LTSS includes a broad range of care and support services for persons who need assistance because of chronic illnesses, physical or mental disability. In general, LTSS consists of personal assistance with activities of daily living (ADLs) or instrumental activities of daily living (IADLs).

While Medicare, the federal program covers most of the acute medical care for the elderly and disabled, it only tangentially covers LTSS. Medicaid is the only public health program that covers LTSS, but it is a safety-net program that offers coverage to only people with limited financial resources. Therefore, the majority of the LTSS needs remain uninsured and most elderly who need LTSS rely on unpaid help from friends or family members. People receive assistance for LTSS in a variety of settings. About $80 \%$ of people receiving such care live in a community setting and the rest $20 \%$ obtain care in the institutions (such as nursing homes). Family caregivers (family members and friends, generally unpaid) are the first line of assistance for most people with LTSS needs those who live in the community. About two out of three of older people who receive LTSS at home receive care exclusively from family members and more than a third of these people have substantial care needs. Approximately 40 million family caregivers offered unpaid care to adults with LTSS needs in 2013 and the estimated economic value of such care was $\$ 470$ billion [3]. Elderly those who live in the community may also receive "in-home care" such as home health aides as well as in the community-based residencies (assisted living facilities, private homes) that offer LTSS. In contrast, elderly with severe functional or cognitive limitations who require around-the-clock assistance often live in institutional settings such as nursing homes, residential care facilities. Over the last two decades the growth in assisted living has been associated with a dramatic decline in nursing home use and the market for nursing home care by the elderly [4]. The national mean occupancy rates declined from $93 \%$ in 1977 to $83 \%$ in 2003 [5,6]. Although it is difficult to have precise estimates for assisted living facilities, one study has estimated that nationwide approximately, there were 11,276 assisted living facilities with 839,746 units in 2007 [7] while approximately 16,100 nursing home facilities with 1.7 million beds in 2004 [8]. Therefore, it is clear that elderly those who need complex array of LTSS receive care from informal as well as formal sources (home health, assisted living or nursing home care).

A major challenge facing the country is how best (i.e. find a balance between informal and formal LTSS) to meet the growing needs for LTSS in most homelike- setting and least institutional setting and achieve best possible health outcomes. To address this challenge the evaluation of relative effectiveness of the use of informal and formal LTSS on care recipients' physical, emotional and mental health outcomes is imperative. A key to producing insights into the balance between the use of informal and formal care would depend on 
understanding how these two types of care benefit patients' physical and mental health outcomes. Any potential public health policy that aims to address the growing needs for LTSS and to estimate consequences of any such policy change on health outcomes requires a causal analysis of the relationship between different types of LTSS use and health outcomes. This is because causal inference is implicitly or explicitly embedded in any public health policy formulation which must be addressed while using observational data [9]. Without this evaluation, informed public policy formulation relating to the optimal use of both types of LTSS achieving positive health outcomes will be unclear. The goals of this brief review article are 1) to investigate existing evidence on the benefits of the use of informal and formal LTSS on the older adults' physical and mental health outcomes and 2) potential areas for future research on empirical evidence of the effectiveness of the use of informal and formal LTSS influencing care recipients' health outcomes.

\section{LTSS Use and Outcomes: Current Evidence}

The literature linking the LTSS use and care recipients' health outcomes is sparse and limited. Existing research predominantly focuses on how providing unpaid (or in combination of both unpaid and paid care) LTSS care impact caregivers' health and wellbeing and potential interventions to improve health outcomes for caregivers. This literature includes older adults with or without cognitive impairments who need LTSS for their daily living within the community setting. For example, a great deal of research has shown that caregiving for individuals with dementia can negatively impact caregivers' outcomes such as burden, distress, physical morbidity and mortality [10-13] Studies also found both positive and negative impacts on caregiving on caregivers physical and mental health outcomes [14,15]. Furthermore, given the expansion of home and community-based services (HCBS) during the past two decades many demonstration studies examined psychosocial outcomes such as life satisfaction, social activity, social interaction of information caregivers under HCBS [16]. Another study reported the positive effects of adult day care services, a low-cost care management intervention on caregiver well-being and quality of life using a quasi-experimental design [17].

The impact of informal caregiving on the labor market outcomes of primary caregivers is another area that has received attention in caregiving literature. Although this evidence is pervasive and wideranging, lost earnings, changes in job schedule due to caregiving responsibilities are the most commonly stated outcomes found in the literature. For example, negative association between informal care and employment is supported by the theory of opportunity costs $[18,19]$, other studies refute the possible association between caregiving and caregivers' labor market outcomes [20]. The endogeneity of the choice of informal care makes the association between caregiving and employment outcomes particularly difficult. However, the availability of longitudinal data and sophisticated statistical models enable researchers to establish causal relationship between caregiving and labor market outcomes. But an important point here to note that the impacts of informal caregiving are being examined from a caregiver's perspective not patients who receive the care.

Although the primary motivation for using different types of LTSS is centered solely on the costs, it is important to know how informal and different types of formal LTSS impact patients' physical and mental health outcomes for appropriate policy purposes. Increasing prevalence of ADRD combined with longer life expectancies; strained federal budgets have led policy makers to advocate for increased supply of informal care from friends and family members. But the supply of informal care will be restricted in light of demographic transition, changes in family structure. Recent research offers limited evidence on possible interdependencies between informal and formal LTSS and patient characteristics that impact longitudinal changes in LTSS use $[21,14]$. However, this evidence does not suggest relative effectiveness of two types of LTSS on physical and menal health outcomes of care recipients. More importantly, without the knowledge of how these two types of LTSS impact patients' health, any successful public policy encouraging informal care for lowering costs of LTSS will not be effective in improving patients' health outcomes. Specifically, relative impacts of the use of informal and various formal LTSS on patients' physical and mental health outcomes, social engagement or life satisfaction are some of the important measures that need to be thoroughly investigated to establish evidence on potential benefits of different types of LTSS from patients perspectives.

One recent review of literature compares the relative effects of home and community based services (through paid assistance) and nursing home services on the outcome trajectories of older adults received formal LTSS [22]. This study has found that older adults served in nursing home or assisted living facilities did not differ in physical function, cognition, mental health, and mortality outcomes. Although evidence was mixed, this type of study can address concerns of many state governments where policy makers have increasingly prioritized home and community based services over the institutional nursing home care to restrain LTSS costs. However, this line of research does not address the relative impacts of unpaid care and different types of paid LTSS on the outcome trajectories of care recipients to assess the potential benefits from policies encouraging or supporting family based informal care.

\section{Future research on effectiveness of informal and formal LTSS and care recipients' outcomes}

The first step to investigate how different types of LTSS impact patients' outcomes is to identify a set of physical, mental and emotional health measures that capture overall health and well-being of care recipients. However, investigating this research question largely depends on data availability that researchers can utilize in retrospective study designs or ability to link multiple observational datasets to estimate the effects different types of LTSS on care recipients' health outcomes. The list of relevant outcome measures, family structure and availability of informal care, change in formal care market characteristics and potential data availability for future research on this topic are discussed below.

\section{Outcome measures}

Health and healthcare utilization measures are typically selected because of likely responsiveness to the skills, training and resources available to care providers. The economic reason in favor of home based care is largely based on consumer preference that individuals prefer to receive LTSS in the most homelike setting and least institutional setting. Outcomes of interest include measures of physical and mental health, social engagement, emotional health including life satisfaction, and self-efficacy. Figure 1 describes the trajectory of outcome measures that will be important to investigate the comparative effectiveness of informal and formal LTSS on physical and mental health status for older adults. 


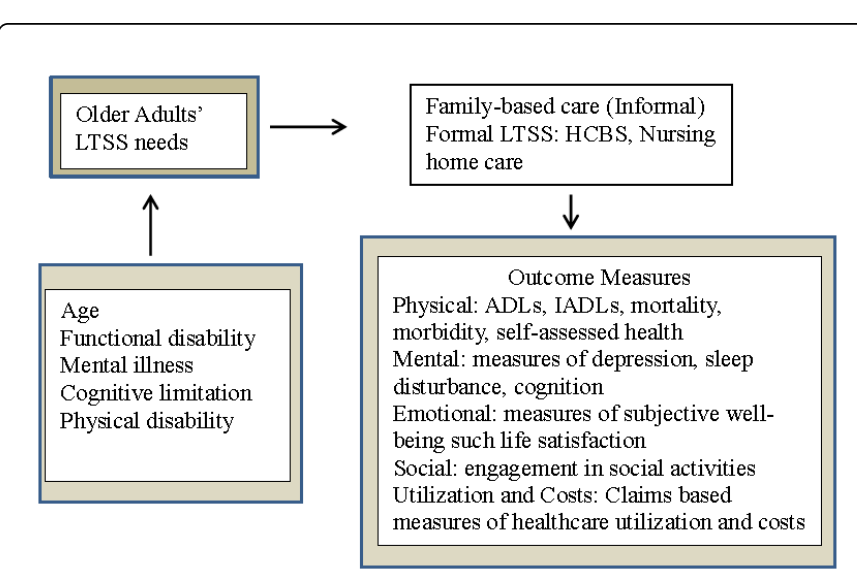

Figure 1: The trajectory of outcome measures (HCBS: Home and Community-based Services).

Physical health: Some important measures of physical health status can include progression of functional disability (new onset or progression of ADLs, IADLs, walking several blocks, or across room, several flights of stairs etc.), self-assessed health (for people with cognitive impairment, proxy or caregiver reported health status), mortality coexistence of multimorbidity (measured by the number of chronic conditions).

Mental health: The Center for Epidemiologic Studies Depression Scale (CES-D) is the most widely used self-report measure of depressive symptoms that can be used to examine mental health status [23]. Literature has suggested the appropriate cut-off points acceptable for defining clinical depression in the general population, especially older adults [24]. In addition to the CES-D score, other potential measures that may include physical manifestation of possible depression such as difficulty in falling sleep, sleep disturbance can also be used as measures of mental health status.

Social engagement: Social engagement refers to the social connection and participation in social activities. Social engagement and its relationship to formal LTSS provision, especially in assisted living facilities, have been shown to be positive [25]. Social engagement can be assessed with measures of social activity frequency, size of social networks and perceived social support [26]. For example, frequency of social activity can be assessed by how often a person is engaged in common types of activities such as attending the church, community volunteer activities, social visits with friends/relatives etc. during a specified period of time. A composite measure of social engagement index can also be constructed including different dimensions of social engagement [27].

Emotional health: Subjective well-being measure has gained popularity as relevant outcome measures in health economics and outcome research studies because its connection with the utility maximization concept which is a central idea of economics. Subjective well-being can be assessed by life satisfaction, happiness such as Diener's measures of life satisfaction, which is a validated and reliable measure of subjective well-being [28,29].

Mortality: Mortality can be used to examine how informal versus formal LTSS care affect death incidence among individuals receiving both types of care.
Healthcare utilization and costs: Self-reported utilization and costs data can be used from population based surveys such as Health and Retirement Study (HRS). The survey reported data will allow researchers explore a wide array of utilization based measures including preventive healthcare use, inpatient (hospital), outpatient visits, and medical expenditure.

Claims-based utilization and cost measures: To address potential recall bias in self-reported measures in the survey, researchers can use the CMS administrative claims data which will offer accurate measures of healthcare utilization and spending. The CMS data can be used in conjunction to the survey data to validate potential impacts of informal and various types of formal LTSS use of physical and mental health outcomes of care recipients. For example, the HRS data can be linked to the CMS administrative claims data after obtaining the restricted data use agreement from the HRS. This linkage will allow researchers unprecedented opportunities to address a broad array of research questions related to effectiveness of informal and various types of formal LTSS on patients' outcomes and potential implications for public policies.

Data sources: Examination of impacts of informal and formal LTSS care on health outcomes requires access to both population based survey data and /or administrative data from the CMS. In order to estimate these impacts precisely, it is important to ensure that measures of informal and formal LTSS can be linked with care recipients' physical and mental health outcomes. For example, the HRS collects extensive information on informal caregiving, family structure affecting the caregiving choice along with person level information on health outcomes, healthcare utilization. Furthermore, the HRS restricted data sources will allow researchers to link geographic information to the existence of formal LTSS market based on state of residence. The HRS respondents can also be linked with the CMS (both Medicare and Medicaid) claims data to access accurate information on enrollment to Medicare, entitlement status (parts $\mathrm{A} / \mathrm{B} / \mathrm{D}$ ), participation in Medicare managed care plans. Medical care utilization can be extracted from CMS's inpatient and outpatient files and pharmacy claims can also be used to collect information on prescription medical use. Different types of cost of care measures are also available in CMS and researchers can utilize those measures based on particular research question at hand.

LTSS market structure: To examine effectiveness of informal and formal LTSS care on patients' health outcomes, it is important to have the data on the local LTSS market because the market for LTSS has been changed remarkably due to the growth of the assisted living industry in the past two decades. There are publicly available resources such as Area Resource File (ARF) which offers local and county level information on home health agencies or assisted living facilities. Newly developed NIC-MAP data source provides comprehensive information on market level data on independent living, assisted living, memory and nursing home care for 100 major US cities (http:// www.nicmap.org). NIC-MAP data are at the facility level, including number of beds, rental versus purchase, profit versus non-profit status, occupancy rates, and number of years in business. Another way of gathering assisted living facility data is by contacting state Medicaid office or state licensure office or other responsible agencies and request information on registered assisted living facilities. Nursing home data can be obtained from two sources: the first source would be the use of the Online Survey Certification and Reporting (OSCAR) system which lists all Medicaid and Medicare certified facilities. The second source of the nursing home data would be the facility-level case mix measures 
that can be generated using the Minimum Data Set (MDS) available from the Brown University's “Long-Term Care: Facts on Care about the US (LTCfocUS) (http://ltcfocus.org/).

\section{Conclusion}

The current review documents an important public health issue that our nation is facing for protecting health and well-being of our elderly population. An optimal balance between informal and various types of LTSS based on relative effectiveness on patients' health outcomes remains a critical public health challenge in the US. Finding this optimal balance will depend on the assessment of relative benefits of informal and formal LTSS care so that appropriate and informed policies can be formulated in moving forward. The current article discusses the importance of this research question, relevant measures of patient level outcomes, and data resources. Future research should utilize existing resources to address this important research question so that appropriate public policy can be formulated to address the growing challenges to meet the demand for long-term care for the elderly in future years.

\section{References}

1. Congressional Budget Office (2013) The rising demand for long-term services and supports for the elderly.

2. http://ltccommission.org/ltccommission/wp-content/uploads/2013/12/ Commission-on-Long-Term-Care-Final-Report-9-26-13.pdf

3. Fox-Grage W (2017) Medicaid: A Last Resort for People Needing Long Term Care and Support Services. AARP Public Policy Institute.

4. Bishop CE (1999) Where are the missing elders? The decline in nursing home use, 1985 and 1995. Health Affairs 18: 146-155.

5. Strahan GW (1997) An Overview of Nursing Homes and Their Current Residents: Data from the 1995 National Nursing Home Survey. Rockville, MD: National Center for Health Statistics, Centers for Disease Control and Prevention, US Department of Health and Human Services.

6. Gibson MJ, Gregory SR, Houser AN, Fox-Grade W (2004) Across the States: Profiles of Long-Term Care (6th edn), DC: AARP Public Policy Institute, Washington.

7. Stevenson DG, Grabowski DC (2010) Sizing up the market for assisted living. Health Affairs 29: 35-43.

8. Jones AL, Dwyer LL, Bercovitz AR, Strahan GW (2009) National Nursing Home Survey: 2004 Overview. Hyattsville, MD: National Center for Health Statistics. Vital Health Statistics 13: 167.

9. Glass TA, Goodman SN, Hernán MA, Samet JM (2013) Causal inference in public health. Ann Rev Pub Health 34: 61-75.

10. Schulz R, O'Brien A, Czaja S, Ory M, Norris R, et al. (2002) Dementia caregiver intervention research: in search of clinical significance. Gerontol 42: 589-602.

11. Schulz R, Belle SH, Czaja SJ, McGinnis KA, Stevens A, et al. (2004) Longterm care placement of dementia patients and caregiver health and wellbeing. JAMA 292: 961-967.
12. Schulz R, Martire LM (2004) Family caregiving of persons with dementia: prevalence, health effects, and support strategies. Am J Ger Psych 12: 240-249.

13. Schulz R, Beach SR (1999) Caregiving as a risk factor for mortality: the caregiver health effects study. JAMA 282: 2215-2219.

14. Basu R, Hochhalter AK, Stevens AB (2015) The impact of the REACH II intervention on caregivers' perceived health. J Appl Gerontol 34: 590-608.

15. Goren A, Montgomery W, Kahle-Wrobleski K, Nakamura T, Ueda K (2016) Impact of caring for persons with Alzheimer's disease or dementia on caregivers' health outcomes: findings from a community based survey in Japan. BMC Geriatrics 16: 122.

16. Grabowski DC (2006) The cost-effectiveness of non-institutional longterm care services: Review and synthesis of the most recent evidence. Med Care Res Rev 63: 3-28.

17. Gitlin LN, Reever K, Dennis MP, Mathieu E, Hauck WW (2006) Enhancing quality of life of families who use adult day services: Short-and long-term effects of the adult day services plus program. Gerontologist 46: 630-639.

18. Becker GS (1965) A Theory of the Allocation of Time. Econ J 493-517.

19. Pezzin LE, Kemper P, Reschovsky J (1996) Does publicly provided home care substitute for family care? Experimental evidence with endogenous living arrangements. J Hum Res 31: 650-676.

20. Lilly M, Laporte A, Coyte PC (2007) Labor market work and home care's unpaid caregivers: A systematic review of labor force participation rates, predictors of labor market withdrawal, and hours of work. Milbank Quar 95: 641-690.

21. Zhu CW, Torgan R, Scarmeas N, Albert M, Brandt J, et al. (2008) Home health and informal care utilization and costs over time in Alzheimer's disease. Home Health Care Services Quar 27: 1-20.

22. Wysocki A, Butler M, Kane RL, Kane RA, Shippee T, et al. (2015) Longterm services and supports for older adults: A review of home and community-based services versus institutional care. J Aging Soc Pol 27: 255-279.

23. Carleton RN, Thibodeau MA, Teale MJ, Welch PG, Abrams MP, et al. (2013) The center for epidemiologic studies depression scale: a review with a theoretical and empirical examination of item content and factor structure. PloS one 8: e58067.

24. Hays JC, Blazer DG, Gold DT (1993) CESD: Cutpoint or Change Score?. J Am Geriatrics Soc 41: 344-345.

25. Zimmerman S, Scott AC, Park NS, Hall SA, Wetherby MM, et al. (2003) Social engagement and its relationship to service provision in residential care and assisted living. Soc Work Res 27: 6-18.

26. Kruege KR, Wilson RS, Kamenetsky JM, Barnes LL, Bienias JL, et al. (2009) Social engagement and cognitive function in old age. Exp Aging Res 35: 45-60.

27. Park NS (2009) The relationship of social engagement to psychological well-being of older adults in assisted living facilities. J Appl Gerontol 28: 461-468.

28. Diener ED, Emmons RA, Larsen RJ, Griffin S (1985) The satisfaction with life scale. J Personal Assess 49: 71-75.

29. Pavot W, Diener E (1993) Review of the Satisfaction With Life Scale. Psychological Assessment 5: 164-172. 